\title{
Neoadjuvant chemotherapy choices from population selection to regimen design: the St. Gallen International Expert Consensus Conference 2021
}

\author{
Qianqian Guo', Qianjun Chen ${ }^{2}$ \\ ${ }^{1}$ Guangdong Provincial Academy of Chinese Medical Sciences, Guangdong Provincial Hospital of Chinese Medicine and The Second Clinical \\ College, Guangzhou University of Chinese Medicine, Guangzhou, China; ${ }^{2}$ Breast Department, Guangdong Provincial Hospital of Chinese Medicine \\ and The Second Clinical College, Guangzhou University of Chinese Medicine, Guangzhou, China \\ Correspondence to: Professor Qianjun Chen. Breast Department, Guangdong Provincial Hospital of Chinese Medicine and The Second Clinical \\ College, Guangzhou University of Chinese Medicine, Guangzhou, China. Email: cqj55@163.com.
}

Received: 28 June 2021; Accepted: 26 July 2021; Published: 31 July 2021.

doi: $10.21037 /$ tbcr-21-14

View this article at: https://dx.doi.org/10.21037/tbcr-21-14

Neoadjuvant chemotherapy is an important treatment strategy for early breast cancer, especially for human epidermal growth factor 2 positive (HER2+) and triple-negative breast cancer. In the past, neoadjuvant chemotherapy was mainly used in conjunction with surgical interventions, in which the locally advanced breast cancer is reduced in size and extent through application of neoadjuvant chemotherapy and downstage surgery. Another application was in patients with large tumors for whom undergoing breast-conserving surgery is aesthetically undesirable, or in whom positive lymph nodes could become negative after neoadjuvant chemotherapy, thus making sentinel lymph node biopsy a possibility (1). Previous neoadjuvant studies have shown the pre-operative therapy is equivalent to adjuvant therapy, meaning that the same regimen used before surgery compared with postoperative use has no effect on disease-free survival (DFS) and overall survival (OS) (2); therefore, most of the neoadjuvant treatments are considered for the purpose of surgery at that time.

With the development of neoadjuvant therapy, a modern neoadjuvant strategy will provide better postoperative adjuvant escalation strategies to those non-pathological complete response (pCR) who have been estimated to have a poor prognosis after undergoing neoadjuvant therapy $(3,4)$. These new strategies will improve the overall prognosis of the neoadjuvant chemotherapy population. Therefore, many international guidelines have selectively added the specifically adapted population of HER2+/triple-negative breast cancer patients into neoadjuvant therapy guidelines (5-7). The selection of which population should receive these regimens and their design have become complicated and controversial. The 2021 St. Gallen Expert Consensus Meeting has discussed relevant issues related to neoadjuvant therapy, especially for HER2+/triple-negative breast cancer.

\section{Should patients assessed before surgery and who require adjuvant chemotherapy undergo neoadjuvant chemotherapy?}

The 2021 St. Gallen Expert Consensus Conference had two questions related to this issue. The first question was, "in some countries, guidelines recommend neoadjuvant treatment for all patients that require any chemotherapy based on the diagnostic biopsy. Is this an appropriate approach?" More than half of the experts (60\%) responded in the negative. The second question was, "can genomic assays on core biopsies be used to select patients with estrogen receptor (ER)-positive and HER2-breast cancer for neoadjuvant endocrine $v s$. chemotherapy?” Most experts (73.58\%) responded in the affirmative (see Table 1). The essence of these two questions is similar: should patients assessed before surgery designated to receive adjuvant chemotherapy undergo neoadjuvant chemotherapy? The difference between these two questions is that the second question depends on genetic tools, while the first is based on traditional clinical pathological characteristics. However, the results of the vote on these two questions 
Table 1 Selection of suitable population for neoadjuvant therapy

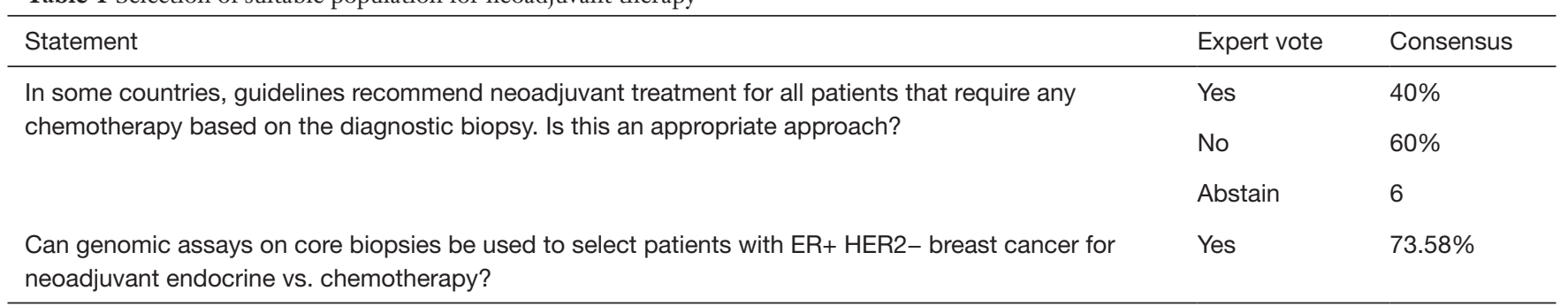

showed opposite trends. How can the response to these two questions inform clinical practice?

Neoadjuvant chemotherapy is a double-edged sword. The advantage includes improving the breast conservation rate, making inoperable patients operable, and preserving the axilla with sentinel lymph node biopsy approach. For triple-negative or HER2 + breast cancer patients with a higher risk of residual disease recurrence (non-pCR population), adjuvant therapy can be applied to improve their survival. However, shortcomings in this approach also exist, including overestimating or underestimating the clinical stage and improperly designing the neoadjuvant chemotherapy regimen, which leads to overtreatment or under (7). A neoadjuvant treatment should meet the following two criteria to avoid these situations: the indications for chemotherapy can be predicted; and the intensity of chemotherapy can be predicted. However, these criteria are not suitable for neoadjuvant treatment conducted for breast-conserving purposes. Even the neoadjuvant therapy may be treated as overtreatment, the cosmetic effects of breast-conserving surgery can balance these negative effects.

For HER2 - breast cancer, the most commonly used chemotherapy regimens include anthracyclines, taxane, or both. Meanwhile, the commonly used regimens for HER 2+ breast cancer include taxane combined with trastuzumab, high-intensity chemotherapy combined with trastuzumab, and high-intensity chemotherapy combined with anti-HER2 dual-target regimens (trastuzumab plus pertuzumab) $(7,8)$. Therefore, predicting the necessity for neoadjuvant treatment before surgery is not enough; it is also essential to predict what intensity the regimen needs to avoid overtreatment or under treatment. For example, for a HER2 + patient with a tumor size of 1.1 centimeters, it can be determined that the patient should receive chemotherapy after surgery, but it is difficult for us to determine the intensity of the chemotherapy regimen. It is still unclear if we should choose the paclitaxel and trastuzumab regimen, as indicated by the adjuvant paclitaxel and trastuzumab (APT) study (9), or a regimen of adriamycin/cyclophosphamide (AC) followed by docetaxel/trastuzumab/pertuzumab (THP) (10). Sixty percent of the experts indicated no for the first question, indicating it is unreasonable for the patients to receive a neoadjuvant treatment based on the adjuvant chemotherapy prediction before the surgery.

Genetic testing tools, including Oncotype DX recurrence score and MammaPrint, have been proven to predict the benefit of adjuvant chemotherapy for hormone receptor (HR)-positive and HER2- breast cancer (11-13). Additionally, retrospective studies have reported that the high Oncotype DX recurrence score may be related to the higher pCR rate of neoadjuvant chemotherapy (14). Nevertheless, genetic testing tools only solve the problem of predicting whether HR+HER2 - patients need chemotherapy but do not tell us what intensity of chemotherapy is needed for a high-scoring genetics patient. For HR+HER2- breast cancer patients who require chemotherapy, if the lymph nodes are negative, the regimen is usually based on alkylating agents and taxane without an anthracyclines. On the contrary, for high-risk tumors with multiple lymph node involvements, a high-intensity regimen of anthracycline combined with a taxane may be preferable (15). Therefore, genetic testing tools cannot be used for neoadjuvant chemotherapy for HR+HER2- breast cancer (16).

In China, the practitioner does not apply the neoadjuvant chemotherapy based on the preoperative prediction for receiving adjuvant chemotherapy, and most guidelines and consensus think that it should be according to the clinical treatment needs and be guided by the purpose of treatment. The three reasons for choosing neoadjuvant treatment are: downgrading inoperable breast cancer to operable breast cancer; downgrading the stage to be available for breastconserving surgery; obtaining drug sensitivity information to guide the subsequent treatment for prognosis improvement (17-19). Currently, it is recognized that the populations who obtain sensitive information to guide 
Table 2 The "standard" regimen for neoadjuvant therapy

\begin{tabular}{|c|c|c|}
\hline Statement & Expert vote & Consensus \\
\hline \multicolumn{3}{|l|}{$\begin{array}{l}\text { More than } 10 \text { years ago, the national agencies proposed pCR as a surrogate end point for drug approval in } \\
\text { early stage breast cancer. Based on experience to date, you would say that }\end{array}$} \\
\hline Regimens that have a remarkable improvement in pCR rates ( $50 \%$ higher than control) should be standard & Yes & $10.17 \%$ \\
\hline \multicolumn{2}{|l|}{$\begin{array}{l}\text { Neoadjuvant pCR rate is encouraging but "standard" regimens should only be defined based on longer-termYes } \\
\text { EFS or OS endpoints }\end{array}$} & $83.05 \%$ \\
\hline
\end{tabular}

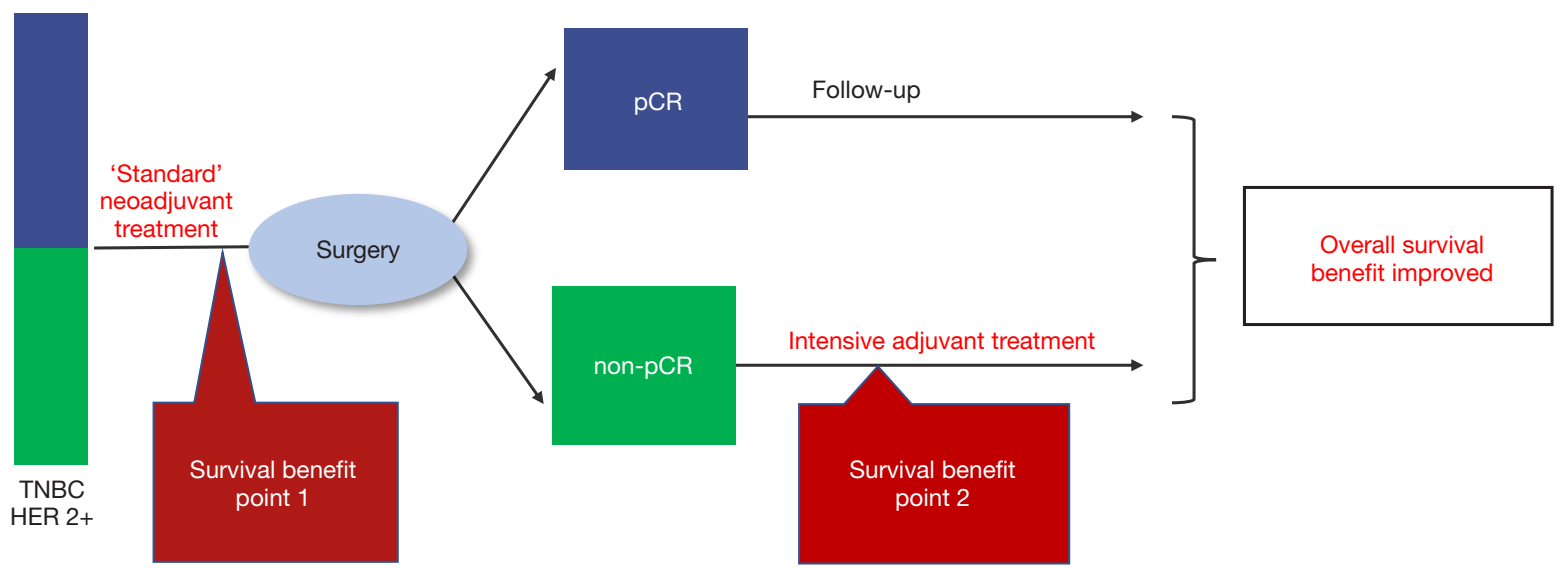

Figure 1 A flowchart of the survival benefit conferred from the neoadjuvant treatment strategy. TNBC, triple-negative breast cancer; HER2+, human epidermal growth factor 2 positive; pCR, pathological complete response.

follow-up treatment are mainly triple-negative and HER2+ patients $(3,4)$. However, in Chinese clinical practice, even triple-negative and HER2 + subtypes cannot be used as an only reference for the selection of neoadjuvant therapy (20), which is consistent with the opinion from the $2021 \mathrm{St}$. Gallen consensus.

\section{Which regimen is the "standard" regimen for neoadjuvant chemotherapy?}

Although different international guidelines have slight differences concerning which population is suitable for neoadjuvant therapy $(7,8,15)$, these populations still have a common feature, nonmetastatic invasive breast cancer. Given this, the purpose of neoadjuvant therapy may be different, but the basic purpose of systemic treatment for nonmetastatic invasive breast cancer is the same: reducing the risk of distant recurrence and improve survival (21). With this established, we can discuss other purposes of applying neoadjuvant therapy, including surgical problems, especially for breast-conserving and axillary surgeries. In this vote, $83.05 \%$ of the experts believed a regimen with long-term event free survival (EFS) or OS evidence can be defined as a "standard" regimen (see Table 2). However, which regimen has the most proven survival benefit evidence has yet to be determined. Only adjuvant therapy has primary survival end points, including DFS, invasive diseasefree survival (iDFS), and OS. Therefore, the "standard" regimen of neoadjuvant therapy should be equivalent or similar to the adjuvant chemotherapy regimen $(6,16)$.

There is a second reason why the standard neoadjuvant regimen is equivalent to the adjuvant chemotherapy regimen. The OS benefits of neoadjuvant on systemic treatment come from a two-stage systemic treatment: the survival benefits conferred by neoadjuvant therapy and the survival benefits conferred by postoperative systemic intensity treatment for the non-pCR population after neoadjuvant therapy (see Figure 1). The latter non-pCR population is the population that has been screened by the "standard" neoadjuvant chemotherapy regimen $(3,22)$. In 
other words, the reported postoperative intensity studies only enrolled the participants with a "standard" neoadjuvant therapy regimen instead of other regimens, even in cases with a high pCR rate reported, including platinum-added regimens.

According to the above analysis, a "standard" neoadjuvant regimen should meet the following two characteristics: the regimen has evidence for improving the survival and the non-pCR information has value in guiding the postoperative adjuvant escalation strategy. Existing research suggests that related studies of neoadjuvant chemotherapy regimens for postoperative adjuvant intensive treatment are derived from "standard" adjuvant chemotherapy regimens. Therefore, the "standard" neoadjuvant design should be based on adjuvant chemotherapy.

In China, most experts (63\%) agree that pCR is a prognostic surrogate endpoint, and neoadjuvant treatment with a high expected $\mathrm{pCR}$ rate may improve the prognosis of patients. Therefore, most experts believe that standard adjuvant therapy regimen (including anti-HER2 therapy) can be used as an alternative regiment to neoadjuvant therapy, but it is not necessarily the preferred one (17). As such, the regimen design for neoadjuvant chemotherapy will include the non-adjuvant regimen, for example, apply the anthracycline and taxane (AT) regimen for HER2breast cancer and design the THP regimen for HER2+ breast cancer $(8,18)$. This opinion is not consistent with the opinion from the 2021 St. Gallen consensus; furthermore, some Chinese experts also do not agree with this point of view (19).

\section{Issues in the design of neoadjuvant therapy regimen for HER2+ breast cancer}

For the HER2+ breast cancer regimen design, the 2021 St. Gallen International Breast Cancer Conference focused on one question, "which patients can be exempted from anthracycline?" As mentioned, the "standard" neoadjuvant regimen design should be based on adjuvant chemotherapy, and HER2+ breast cancer is no exception. The adjuvant systemic treatment for HER2 + breast cancer is based on anti-HER2 targeted therapy combined with chemotherapy, including anthracycline-containing regimens and non-anthracycline-containing regimens. The most critical regimen is taxane combined with carboplatin or anthracycline. From the perspective of survival improvement, anthracycline, taxane, and combined with anti-HER2 targeted therapy have more supporting evidence and have been repeatedly verified by several phase III clinical studies (23-25). However, the evidence for the combination of taxane and carboplatin only comes from one phase III clinical study (25). Anthracycline and taxane combined with anti-HER2 targeted therapy should be the preferred regimen for neoadjuvant treatment for HER2+ breast cancer.

It is now common to subtract systemic chemotherapy for HER2+ breast cancer in the era of anti-HER2 targeting. For some HER2+ breast cancer patients, taxane combined with trastuzumab can already obtain significant survival benefits (9). Studies have demonstrated that anthracycline could be exempted entirely in neoadjuvant therapy $(26,27)$. Meanwhile, another study has illustrated that anthracycline is still an important and indispensable drug even in the era of anti-HER2 dual-target therapy (28). In summary, which patients can have anthracyclines be omitted from their regimen is an important clinical question for HER2+ breast cancer patients.

Some Chinese experts believe anthracycline can be avoided in patients with a high local tumor burden because these patients need to receive anti-HER2 targeted therapy earlier to reach tumor downgrading. If we design an anthracycline-containing regimen, the anti-HER2 targeted therapy should not be combined with anthracycline simultaneously due to adverse events; rather, it should be applied sequentially after anthracycline (25), although these patients are then unable to receive their anti-HER2 targeted therapy as early as possible. In the 2021 St. Gallen expert vote, $84.62 \%$ of the experts indicated that anthracycline is unnecessary in managing patients with stage II nodenegative HER2+ breast cancer who are receiving taxanebased chemotherapy and anti-HER2 antibodies. Also, $53.85 \%$ thought that anthracycline must be used in nodepositive patients. For node-negative stage II/III breast cancer, more than half of the experts were willing to use the THP or (docetaxel/carboplatin/trastuzumab/pertuzumab) TCbHP regimen, but in lymph node-positive HER2+ breast cancer, $36.37 \%$ of the experts were willing to use the THP or TCbHP plan (see Table 3). This tendency is contrary to some of the Chinese experts' opinions. These results from the St. Gallen consensus indicate that a neoadjuvant regimen for HER2+ breast cancer with a high tumor burden should not be exempted from anthracycline, and experts should not design THP or TCbHP regimens with the earlier use of anti-HER2 dual targets for high tumor burden HER2+ breast cancer patients.

HER2 + breast cancer is a heterogeneous tumor (29). 
Table 3 The neoadjuvant treatment regimen for HER2+ breast cancer patients

\begin{tabular}{|c|c|c|}
\hline Statement & Expert vote & Consensus \\
\hline & No & $84.62 \%$ \\
\hline & Abstain & 7 \\
\hline \multirow{2}{*}{$\begin{array}{l}\text { Are anthracyclines necessary in management of stage II, node-positive HER2+ breast cancer in patients } \\
\text { receiving taxane-based chemotherapy and anti-HER2 antibodies? }\end{array}$} & Yes & $53.85 \%$ \\
\hline & Abstain & 7 \\
\hline \multicolumn{3}{|l|}{$\begin{array}{l}\text { In standard neoadjuvant treatment of HER2 positive breast cancer with clinically positive axillary lymph } \\
\text { nodes, the preferred regimen includes taxane/trastuzumab combined with: }\end{array}$} \\
\hline Pertuzumab & Yes & $12.73 \%$ \\
\hline Abstain & & 4 \\
\hline \multicolumn{3}{|l|}{$\begin{array}{l}\text { In standard neoadjuvant treatment of HER2 positive breast cancer with stage II/III clinically negative axillary } \\
\text { lymph nodes, patients should receive a regimen including taxane/trastuzumab combined with: }\end{array}$} \\
\hline Pertuzumab & Yes & $23.64 \%$ \\
\hline Pertuzumab and platinum agent & Yes & $27.27 \%$ \\
\hline Anthracycline-based & Yes & $12.3 \%$ \\
\hline Anthracycline-based and pertuzumab & Yes & $35.55 \%$ \\
\hline
\end{tabular}

With few exceptions, spontaneous tumors generally originate from a single cell. However, most human tumors show surprising heterogeneity, and tumor heterogeneity is positively correlated with the evolution time of the tumor in the body (30), with the tumor burden being positively correlated with this time. Generally, the greater the tumor burden, the greater the potential for heterogeneity. Some solutions studied to solve the heterogeneity of HER2+ breast cancer include the HER2 cell clones, especially the HR-HER2 cell clone. In the future, it may be possible to use drugs, including DS8201 (31). However, the current clinical practice still mainly relies on traditional chemotherapy drugs, including anthracycline and taxane.

For the clinical practice in China, the design of the HER2 + breast cancer regimen is based on anti-HER2 dual target treatment. However, in the design of chemotherapy regimen, experts at the 2021 St. Gallen meeting tend to decide whether to exempt anthracycline based on tumor burden (lymph node status) classification, while the exemption of anthracycline is the mainstream design applied in China (8).

\section{Design of neoadjuvant chemotherapy for triple- negative breast cancer}

For designing a regimen for triple-negative breast cancer, the 2021 St. Gallen meeting mainly discussed two questions: (I) should platinum be added to the anthracycline combined with taxane? (II) can the targeted therapy of immune checkpoint inhibitor programmed cell death 1/ programmed death-ligand 1 (PD-1/PD-L1) be designed into a neoadjuvant regimen?

For the first issue, $60.38 \%$ of experts at the meeting opposed platinum being added to the anthracycline/taxane (see Table 4). According to the CREAT-X study, for the triple-negative breast cancer patients who are receiving 
Table 4 Design of neoadjuvant chemotherapy for triple-negative breast cancer

\begin{tabular}{|c|c|c|}
\hline Statement & Expert vote & Consensus \\
\hline \multirow{2}{*}{$\begin{array}{l}\text { Should patients receiving neoadjuvant chemotherapy also receive carboplatin when given standard "dose- } \\
\text { dense AC and paclitaxel" or other cyclophosphamide/anthracycline/taxane-type regimens? }\end{array}$} & No & $60.38 \%$ \\
\hline & Abstain & 5 \\
\hline $\begin{array}{l}\text { Should patients receiving standard neoadjuvant chemotherapy also receive PD1/PDL1 targeted treatment } \\
\text { with an immune checkpoint inhibitor? }\end{array}$ & Yes & $9.62 \%$ \\
\hline
\end{tabular}

neoadjuvant therapy, the survival benefits from systemic treatment come from neoadjuvant therapy and adjuvant capecitabine therapy in non-pCR populations (3). It should be noted that $95.3 \%$ of the patients in the CREAT-X study received neoadjuvant chemotherapy with anthracycline and taxane instead of anthracycline and taxane combined with platinum. If platinum is incorporated into the neoadjuvant regimen, does the non-pCR patient need capecitabine after surgery? The evidence for an answer to this question is currently limited. If no further treatment is needed for nonpCR patients, the survival benefit evidence is also limited. Therefore, neoadjuvant chemotherapy for triple-negative breast cancer should use standard anthracycline combined with a taxane, and platinum should not be routinely added.

Another question is whether the addition of platinum to the anthracycline and taxane should be barred in all neoadjuvant chemotherapies for triple-negative breast cancer. The disadvantages for adding the platinum are as follows: (I) the data on whether adjuvant enhancement provides benefit for a non-pCR situation are limited, (II) the survival data on this regimen are contradictory, and (III) the adverse event frequency is increased. The advantage is that the regimen provides a higher $\mathrm{pCR}$ rate than does anthracycline combined with taxane (32-34). Hence, the disadvantage and advantages of adding platinum should be weighed in clinical practice. For those patients who urgently need the advantage of "high response rate"-or "the population who needs better local control"—adding platinum would be a better choice (35). For example, when $\mathrm{AC}$ followed by a $\mathrm{T}$ regimen designed for inoperable locally advanced breast cancer patients is estimated to have poor efficacy with first applied AC regimen, adding platinum could be an option.

At the 2021 St. Gallen meeting, 90.38\% of experts opposed adding a targeted therapy with immune-checkpoint inhibitor PD-1/PD-L1 in addition to the standard neoadjuvant chemotherapy for triple-negative breast cancer (see Table 4). Some studies have shown that the addition of immune-checkpoint inhibitors to a triple-negative breast cancer chemotherapy regimen may increase the pCR rate $(36,37)$, while some studies have shown it does not (38). However, inducing the immunotherapy sensitivity during the window phase may increase the pCR rate (39). The best timing to receive an immune checkpoint inhibitor in neoadjuvant therapy, the timing and duration of treatment, which neoadjuvant chemotherapy regimen should be used in combination, the appropriate treatment population (especially whether PD-L1 negative cancer patients are appropriate), the long-term outcomes for immunotherapy (including EFS and OS), and the effects of toxicity remain undetermined. Therefore, current guidelines do not recommend adding immune checkpoint inhibitors to the standard regimen of neoadjuvant chemotherapy for triplenegative breast cancer in clinical practice (16). However, patients are encouraged to participate in clinical research (8).

In Chinese clinical practice, the neoadjuvant regimen design for triple-negative includes six to eight cycles with anthracycline and taxane (for example, AC followed by docetaxel or doxetaxel/adriamycin/cyclophosphamide) (17); some guideline points out that the taxane plus with platinum regimen can obtain a higher pCR rate for triple-negative breast cancer but without stage III randomized clinical trial evidence(8). For immunotherapy, it is currently only recommended to participate in strictly designed clinical studies due to the indication has not been approved (16).

\section{Conclusions}

Neoadjuvant chemotherapy is a double-edged sword. It is necessary to select the appropriate population to avoid excessive and insufficient treatment. In clinical practice, adhering to the two necessary conditions to select the neoadjuvant population-that the indications of chemotherapy can be predicted and the intensity of chemotherapy can be predicted-helps to avoid excessive and unacceptable risk. Therefore, performing neoadjuvant 
therapy only based on the prediction that the patient needs adjuvant chemotherapy before surgery is uncalled for, as this will potentially increase the risk of overtreatment in neoadjuvant therapy. Additionally, the selection of neoadjuvant population should also consider the patients' personal opinion, especially those with available breast conservation surgery and lymph node negative patients.

The characteristics of the neoadjuvant population are curable or potentially curable. Therefore, reducing the risk of distant recurrence and improving survival are the basic goals of systemic treatment (including neoadjuvant treatment). Improvement in survival of these patients with systemic treatment entails a benefit from the neoadjuvant phase and postoperative adjuvant systemic treatment for the non-pCR population. Therefore, the design of a "standard" neoadjuvant chemotherapy regimen should not only consider whether the neoadjuvant chemotherapy regimen has evidence of survival benefits (not whether pCR is improved) but also consider whether the non-pCR information "screened" by the regimen can guide postoperative adjuvant escalation strategy and bring survival improvement.

For HER2+ breast cancer, the "standard" neoadjuvant regimen includes anthracycline-containing and nonanthracycline-containing regimens. The 2021 St. Gallen experts prefer to design an anthracycline-containing regimen for patients with a high tumor burden with positive lymph nodes. However, a non-anthracycline-containing regimen can be considered for patients with negative lymph nodes. No evidence is available concerning survival benefits in the neoadjuvant phase for triple-negative breast cancer patients undergoing platinum or immune-checkpoint inhibitor regimens over standard anthracycline taxanebased regimens. It is even less clear whether those nonpCR populations "screened" through these regimens have a postoperative escalation strategy. Consequently, most experts do not recommend the addition of platinum or immune checkpoint inhibitors to neoadjuvant therapy for triple-negative breast cancer.

Furthermore, it is necessary to balance potential benefits and side effects in neoadjuvant chemotherapy regimens; for example, the decision to add the platinum regimen to the anthracycline taxane based regimens for triple-negative breast cancer patients. Other consideration also includes the cost-effectiveness, especially in the application of antiHER2 dual target neoadjuvant treatment for node-negative HER2 + breast cancer.

The research of neoadjuvant chemotherapy has made rapid progress, especially in the field of triple-negative breast cancer and HER2 + breast cancer. However, the research endpoint for most previous studies is still the pCR rate, which is insufficient for curable or potentially curable neoadjuvant chemotherapy populations. For instance, if the triple-negative breast cancer patients received the taxane plus platinum in the neoadjuvant treatment, it is not clear about how to choose the anthracycline or capecitabine adjuvant treatment for the non-pCR population. Therefore, the high pCR regimen developed in the neoadjuvant treatment still needs further research in the future to better guide the clinical practice.

Previous studies mostly based on the traditional immunohistochemistry substitution subtype for research design, however with the development of molecular biology, whether it is possible to design research based on better molecular biological models-such as HER2 + subtype breast cancer-is still not clear. For p95HER2 subtypes, whether the trastuzumab with small molecule tyrosine kinase inhibitors is better than the currently used dual-target approach (trastuzumab and pertuzumab) is still remain doubts.

In current clinical practice, the guidance information for adjuvant escalation strategies is derived from nonpCR population, but studies have shown that even if the pCR is reached, a considerable number of patients will still relapse. Obviously, the adjuvant escalation strategies based on the non-pCR information guidance is an approach that needs further exploration. Whether it can surpass pCR and non-pCR information and use modern molecular biology technology to accurately screen out the population that needs adjuvant escalation treatment is worthy of further research. The development of these technologies will even shake the status of the neoadjuvant chemotherapy strategy itself, especially for those neoadjuvant chemotherapy applied "for the purpose of sensitivity judgment". The use of neoadjuvant chemotherapy to do subtractive research on traditional "standard schemes", immunotherapy drugs, and new antibody-drug-conjugates drugs are also important areas that need attention in the future of neoadjuvant research.

\section{Acknowledgments}

Funding: None.

\section{Footnote}

Provenance and Peer Review: This article was commissioned by the Editorial Office, Translational Breast Cancer Research. The article underwent external peer review. 
Conflicts of Interest: Both authors have completed the ICMJE uniform disclosure form (available at https://dx.doi. org/10.21037/tbcr-21-14). The authors have no conflicts of interest to declare.

Ethical Statement: The authors are accountable for all aspects of the work in ensuring that questions related to the accuracy or integrity of any part of the work are appropriately investigated and resolved.

Open Access Statement: This is an Open Access article distributed in accordance with the Creative Commons Attribution-NonCommercial-NoDerivs 4.0 International License (CC BY-NC-ND 4.0), which permits the noncommercial replication and distribution of the article with the strict proviso that no changes or edits are made and the original work is properly cited (including links to both the formal publication through the relevant DOI and the license). See: https://creativecommons.org/licenses/by-nc-nd/4.0/.

\section{References}

1. NCCN Clinical Practice Guidelines in Oncology, Breast Cancer (Version 3. 2019). 3rd ed: National Comprehensive Cancer Network; 2019:BINV-M, 2 OF.

2. Rastogi P, Anderson SJ, Bear HD, et al. Preoperative chemotherapy: updates of National Surgical Adjuvant Breast and Bowel Project Protocols B-18 and B-27. J Clin Oncol 2008;26:778-85.

3. Masuda N, Lee SJ, Ohtani S, et al. Adjuvant capecitabine for breast cancer after preoperative chemotherapy. $\mathrm{N}$ Engl J Med 2017;376:2147-59.

4. von Minckwitz G, Huang CS, Mano MS, et al. Trastuzumab emtansine for residual invasive HER2positive breast cancer. N Engl J Med 2019;380:617-28.

5. Guidelines of Chinese Society of Clinical Oncology (CSCO), Breast cancer (Version 1. 2017). People's Medical Publishing House; 2017.

6. Curigliano G, Burstein HJ, Winer EP, et al. De-escalating and escalating treatments for early-stage breast cancer: the St. Gallen International Expert Consensus Conference on the primary therapy of early breast cancer 2017. Ann Oncol 2017;28:1700-12.

7. NCCN Clinical Practice Guidelines in Oncology, Breast Cancer (Version 1. 2021). National Comprehensive Cancer Network; 2021:BINV-M, 1 OF 2.

8. Guidelines of Chinese Society of Clinical Oncology (CSCO), Breast cancer(Version 1. 2021). People's Medical
Publishing House; 2021.

9. Tolaney SM, Guo H, Pernas S, et al. Seven-year followup analysis of adjuvant paclitaxel and trastuzumab trial for node-negative, human epidermal growth factor receptor 2-positive breast cancer. J Clin Oncol 2019;37:1868-75.

10. von Minckwitz G, Procter M, de Azambuja E, et al. Adjuvant pertuzumab and trastuzumab in early HER2positive breast cancer. N Engl J Med 2017;377:122-31.

11. Sparano JA, Gray RJ, Makower DF, et al. Adjuvant chemotherapy guided by a 21-gene epression assay in breast cancer. N Engl J Med 2018;379:111-21.

12. Kalinsky K, Barlow WE, Meric-Bernstam F, et al. First results from a phase III randomized clinical trial of standard adjuvant endocrine therapy (ET) +/chemotherapy (CT) in patients (pts) with 1-3 positive nodes, hormone receptor-positive (HR+) and HER2negative (HER2-) breast cancer (BC) with recurrence score (RS) < 25: SWOG S1007 (RxPonder). SABCS 2021;81:Abstract GS3-00.

13. Piccart $M$, van 't Veer LJ, Poncet C, et al. 70-gene signature as an aid for treatment decisions in early breast cancer: updated results of the phase 3 randomised MINDACT trial with an exploratory analysis by age. Lancet Oncol 2021;22:476-88.

14. Pease AM, Riba LA, Gruner RA, et al. Oncotype DX(®) recurrence score as a predictor of response to neoadjuvant chemotherapy. Ann Surg Oncol 2019;26:366-71.

15. Burstein HJ, Curigliano G, Loibl S, et al. Estimating the benefits of therapy for early-stage breast cancer: the St. Gallen International Consensus Guidelines for the primary therapy of early breast cancer 2019. Ann Oncol 2019;30:1541-57.

16. Korde LA, Somerfield MR, Carey LA, et al. Neoadjuvant chemotherapy, endocrine therapy, and targeted therapy for breast cancer: ASCO guideline. J Clin Oncol 2021;39:1485-505.

17. Group CBCNTE. Expert consensus on neoadjuvant treatment of breast cancer in China. China Oncology 2019:390-400.

18. Qiao J, Liu Z. Expert consensus on neoadjuvant treatment of breast cancer in Henan Cancer Hospital. Chinese Journal of Cancer Prevention and Treatment 2019;26:1872-32.

19. Cai G, Cai Z, Chen Q, et al. Current status and progress of neoadjuvant chemotherapy for breast cancer--main topics and consensus of the Southern Breast Cancer Forum. Chinese Journal of General Surgery 2019;28:1309-21.

20. Chinese Anti-Cancer Association CoBCS. Guidelines and 
standards for diagnosis and treatment of breast cancer by Chinese Anti-Cancer Association. China Oncology 2019;29:609-80.

21. Kaufmann M, von Minckwitz G, Mamounas EP, et al. Recommendations from an international consensus conference on the current status and future of neoadjuvant systemic therapy in primary breast cancer. Ann Surg Oncol 2012;19:1508-16.

22. Tutt ANJ, Garber JE, Kaufman B, et al. Adjuvant olaparib for patients with BRCA1- or BRCA2-mutated breast cancer. N Engl J Med 2021;384:2394-405.

23. Perez EA, Suman VJ, Davidson NE, et al. Sequential versus concurrent trastuzumab in adjuvant chemotherapy for breast cancer. J Clin Oncol 2011;29:4491-7.

24. Perez EA, Romond EH, Suman VJ, et al. Trastuzumab plus adjuvant chemotherapy for human epidermal growth factor receptor 2-positive breast cancer: planned joint analysis of overall survival from NSABP B-31 and NCCTG N9831. J Clin Oncol 2014;32:3744-52.

25. Slamon D, Eiermann W, Robert N, et al. Adjuvant trastuzumab in HER2-positive breast cancer. N Engl J Med 2011;365:1273-83.

26. Schneeweiss A, Chia S, Hickish T, et al. Pertuzumab plus trastuzumab in combination with standard neoadjuvant anthracycline-containing and anthracycline-free chemotherapy regimens in patients with HER2-positive early breast cancer: a randomized phase II cardiac safety study (TRYPHAENA). Ann Oncol 2013;24:2278-84.

27. van der Voort A, van Ramshorst MS, van Werkhoven ED, et al. Three-year follow-up of neoadjuvant chemotherapy with or without anthracyclines in the presence of dual ERBB2 blockade in patients with ERBB2-positive breast cancer: a secondary analysis of the TRAIN-2 randomized, phase 3 trial. JAMA Oncol 202 1;7:978-84.

28. Masuda N, Ohtani S, Takano T, et al. Neoadjuvant therapy with trastuzumab emtansine and pertuzumab in patients with HER2-positive primary breast cancer (a randomized, phase 2 study; JBCRG-20). Ann Oncol 2017;28:v47-8.

29. Risom T, Wang X, Liang J, et al. Deregulating MYC in a model of HER2 + breast cancer mimics human intertumoral heterogeneity. J Clin Invest 2020;130:231-46.

30. Marusyk A, Polyak K. Tumor heterogeneity: causes and consequences. Biochim Biophys Acta 2010;1805:105-17.

31. Nicolò E, Zagami P, Curigliano G. Antibody-drug conjugates in breast cancer: the chemotherapy of the future? Curr Opin Oncol 2020;32:494-502.

32. Sikov WM, Berry DA, Perou CM, et al. Impact of the addition of carboplatin and/or bevacizumab to neoadjuvant once-per-week paclitaxel followed by dosedense doxorubicin and cyclophosphamide on pathologic complete response rates in stage II to III triple-negative breast cancer: CALGB 40603 (Alliance). J Clin Oncol 2015;33:13-21.

33. von Minckwitz G, Schneeweiss A, Loibl S, et al. Neoadjuvant carboplatin in patients with triple-negative and HER2-positive early breast cancer (GeparSixto; GBG 66): a randomised phase 2 trial. Lancet Oncol 2014;15:747-56.

34. Schneeweiss A, Möbus V, Tesch H, et al. Intense dosedense epirubicin, paclitaxel, cyclophosphamide versus weekly paclitaxel, liposomal doxorubicin (plus carboplatin in triple-negative breast cancer) for neoadjuvant treatment of high-risk early breast cancer (GeparOcto-GBG 84): A randomised phase III trial. Eur J Cancer 2019;106:181-92.

35. NCCN Clinical Practice Guidelines in Oncology, Breast Cancer (Version 4. 2021). National Comprehensive Cancer Network; 2021. p. BINV-L, 1 OF 7.

36. Schmid P, Cortes J, Pusztai L, et al. Pembrolizumab for early triple-negative breast cancer. $\mathrm{N}$ Engl J Med 2020;382:810-21.

37. Mittendorf EA, Zhang H, Barrios CH, et al. Neoadjuvant atezolizumab in combination with sequential nab-paclitaxel and anthracycline-based chemotherapy versus placebo and chemotherapy in patients with early-stage triple-negative breast cancer (IMpassion031): a randomised, double-blind, phase 3 trial. Lancet 2020;396:1090-100.

38. Gianni L, Huang CF, Egle D, et al. Abstract GS3-04: Pathologic complete response (pCR) to neoadjuvant treatment with or without atezolizumab in triple negative, early high-risk and locally advanced breast cancer. NeoTRIPaPDL1 Michelangelo randomized study. Abstracts: 2019 San Antonio Breast Cancer Symposium; December 10-14, 2019; San Antonio, Texas.

39. Loibl S, Untch M, Burchardi N, et al. A randomised phase II study investigating durvalumab in addition to an anthracycline taxane-based neoadjuvant therapy in early triple-negative breast cancer: clinical results and biomarker analysis of GeparNuevo study. Ann Oncol 2019;30:1279-88.

doi: $10.21037 /$ tbcr-21-14

Cite this article as: Guo Q, Chen Q. Neoadjuvant chemotherapy choices from population selection to regimen design: the St. Gallen International Expert Consensus Conference 2021. Transl Breast Cancer Res 2021;2:25. 